\title{
THE RADIAL BEHAVIOR OF BLASCHKE PRODUCTS
}

\author{
C. L. BELNA, P. COLWELL AND G. PIRANIAN
}

\begin{abstract}
The paper extends results of G. T. Cargo and of C. L. Belna, F. W. Carroll and G. Piranian on the existence of Blaschke products with prescribed radial limits at a prescribed finite or countable set of points on the unit circle. The extension permits the prescription of the radial cluster set at each point of a prescribed countable set on the unit circle.
\end{abstract}

1. Introduction. G. T. Cargo [2, p. 289] proved that if $\left\{\zeta_{m}\right\}_{1}^{n}$ and $\left\{w_{m}\right\}_{1}^{n}$ are a set of finitely many distinct points on the unit circle $C$ and a set of points in the closure of the unit disk $D$, then some Blaschke product has the radial limit $w_{m}$ at $\zeta_{m}$ for $m=1, \ldots, n$. He asked whether the theorem permits an extension to countably infinite sets $\left\{\zeta_{m}\right\}$ and $\left\{w_{m}\right\}$. Our paper shows that the answer is affirmative, and it goes beyond Cargo's question: it deals not merely with radial limits, but with radial cluster sets.

THEOREM. Let $M=\left\{\zeta_{m}\right\}$ and $\left\{K_{m}\right\}$ denote a countable set of distinct points on $C$ and a sequence of nonempty, closed, connected sets in $D \cup C$. Then some Blaschke product has the radial cluster set $K_{m}$ at $\zeta_{m}$, for $m=1,2, \ldots$

2. The simplest case. We begin with the special case where $M$ consists of the single point $\zeta=1$ and the continuum $K_{1}=K$ consists of a segment on the interval $[0,1]$ of the real axis.

Even in the most general case, our proof will depend on elementary properties of the atomic singular function

$$
A(z)=\exp [(z+1) /(z-1)]
$$

and the composite function $B_{0}=S \circ A$, where

$$
S(w)=\frac{1 / e-w}{1-w / e}=\frac{1-e w}{e-w} .
$$

At the point 1 , the function $A$ has the radial limit 0 and therefore $B_{0}$ has the radial limit $1 / e$. At all other points of $C$, the function $A$ has a radial limit of modulus 1 and therefore $B_{0}$ has a radial limit of modulus 1 . Because $B_{0}$ is a bounded function with a radial limit of modulus 1 almost everywhere on $C$, it is an inner function; because its radial limit is nowhere 0 , it is a Blaschke product.

Received by the editors July 7, 1983. Presented to the AMS in East Lansing, Michigan, on November 13, 1982.

1980 Mathematics Subject Classification. Primary 30D50; Secondary 30D40. 
The zeros of $B_{0}$ are the $1 / e$-points of $A$, in other words, the points $z$ where the real and imaginary parts of $(z+1) /(z-1)$ are equal to -1 and to a multiple of $2 \pi$, respectively. The real part is equal to -1 everywhere on the circular arc $\{|z-1 / 2|$ $=1 / 2, z \neq 1\}$ and nowhere else. We obtain information on the geometric distribution of the zeros of $B_{0}$ on this arc by solving the equation

$$
\left(z_{\nu}+1\right) /\left(z_{\nu}-1\right)=-1+2 \nu \pi i \quad(\nu=0, \pm 1, \pm 2, \ldots):
$$

because the mapping $z \rightarrow(z+1) /(z-1)$ is an involution,

$$
z_{\nu}=\frac{(-1+2 \nu \pi i)+1}{(-1+2 \nu \pi i)-1}=\frac{\nu \pi i}{\nu \pi i-1} .
$$

Obviously, $z_{0}=0$ and $z_{\nu}=1 /(1+i / \nu \pi)(\nu \neq 0)$. It follows that if $\nu \neq 0$, then

$$
z_{\nu}=1-i / \nu \pi-1 /(\nu \pi)^{2}+i /(\nu \pi)^{3}+O\left(\nu^{-4}\right)
$$

simple calculations now show that

$$
\left|z_{\nu}\right|^{2}=\left(1-(\nu \pi)^{-2}\right)^{2}+(\nu \pi)^{-2}+O\left(\nu^{-4}\right)
$$

and therefore $\left|z_{\nu}\right|=1-1 / 2(\nu \pi)^{2}+O\left(\nu^{-4}\right)$.

Suppose that corresponding to some number $\rho(0<\rho<1)$ we have mutilated the Blaschke product $B_{0}$ by suppressing the finitely many factors whose zeros lie outside of the disk $\{|z-1|<\rho\}$. (For the sake of brevity, we shall henceforth speak of suppression of certain zeros rather than suppression of the factors having these zeros.) By virtue of our estimate of $\left|z_{\nu}\right|$, the sum of the distances from the surviving zeros to the unit circle $C$ is approximately

$$
2 \sum_{\nu \pi \rho} 1 / 2(\nu \pi)^{2}=\pi^{-2} \sum_{\nu>\pi / \rho} \nu^{-2} \sim \rho / \pi^{3} .
$$

On the other hand, because the set of suppressed zeros is finite and is symmetric relative to the real axis, the mutilated product has at $z=1$ the same radial limit as $B_{0}$, namely $1 / e$. If corresponding to $\mu=1,2, \ldots$ we obtain $B_{\mu}$ from $B_{0}$ by a finite and symmetric mutilation such that the sum of the distances from the surviving zeros to $C$ is less than $\mu^{-2}$, then the formal infinite product $B=\Pi B_{\mu}$ is again a Blaschke product, and at $z=1$ it has the radial limit 0 . By means of further mutilations, we shall refine our control over the behavior of $B$ on the segment $[0,1)$.

Corresponding to each positive integer $j$ and the integers $k=0,1, \ldots, j-1$, we consider the functions

$$
A_{j k}(z)=\exp \frac{1}{j}\left(\frac{z+1}{z-1}-2 k \pi i\right)=A_{j 0}(z) \exp \left(-\frac{2 k \pi i}{j}\right) .
$$

Because $\left|A_{j k}(0)\right|=e^{-1 / j}$, the functions $A_{j k}$ have the constant modulus $e^{-1 / j}$ on the circular arc $\{|z-1 / 2|=1 / 2, z \neq 1\}$. Clearly, $A_{j k}$ assumes the real value $e^{-1 / j}$ at the points on this arc where the imaginary part of $(z+1) /(z-1)-2 k \pi i$ is a multiple of $2 j \pi$. The function

$$
B_{j k}=\left(1-e^{1 / j} A_{j k}\right) /\left(e^{1 / j}-A_{j k}\right)
$$


has the radial limit 0 nowhere on $C$; therefore it is a Blaschke product. Obviously, its zeros (the $e^{-1 / j}$-points of $A_{j k}$ ) are the zeros $z_{\nu}$ of $B_{0}$ whose indices satisfy the condition $\nu \equiv k(\bmod j)$.

Now let $\left\{\gamma_{k}\right\}$ denote a sequence of numbers in the interval $(0,1)$ such that the segment $K$ is the set of limit points of $\left\{\gamma_{k}\right\}$. For technical reasons, we also require that $\gamma_{1}>e^{-1}$ and that $\log \gamma_{k}$ is rational and $0<\left|\log \gamma_{k+1} / \gamma_{k}\right|<1$ for $k=1,2, \ldots$

We form a periodic sequence $\left\{\nu_{1 i}\right\}_{i=1}^{\infty}$ of density $-\log \gamma_{1}$ and denote by $B_{1}$ the Blaschke product obtained from $B_{0}$ by suppression of all the zeros $z_{\nu}$ except those for which $|\nu| \in\left\{\nu_{1 i}\right\}$. From our earlier discussion it follows that at $z=1$ the function $B_{1}$ has the radial limit $\gamma_{1}$. We can therefore choose a number $r_{1}$ in $(0,1)$ such that

$$
\left|B_{1}(x)-\gamma_{1}\right|<2^{-1} \text { whenever } r_{1} \leqslant x<1
$$

Our next step depends on whether $\gamma_{2}<\gamma_{1}$ or $\gamma_{2}>\gamma_{1}$.

If $\gamma_{2}<\gamma_{1}$, we form a periodic sequence $\left\{\nu_{2 i}\right\}$ of density $-\log \gamma_{2} / \gamma_{1}$ and denote by $B_{2}$ the Blaschke product obtained from $B_{0}$ by suppression of all the zeros $z_{\nu}$ except those for which $|\nu| \in\left\{\nu_{2 i}\right\}$. Then the radial limit of $B_{1} B_{2}$ at $z=1$ is $\gamma_{2}$; moreover, if the initial element of $\left\{\nu_{2 i}\right\}$ is sufficiently large, then $\left|B_{2}(x)-1\right|<2^{-2}$ whenever $0<x<r_{1}$.

If $\gamma_{2}>\gamma_{1}$, we denote by $B_{2}$ the reciprocal of a Blaschke product whose zeros occur in $B_{1}$ and are selected so that $B_{1} B_{2}$ has at 1 the radial limit $\gamma_{2}$. Again, if the sequence of selected zeros begins late enough, then $\left|B_{2}(x)-1\right|<2^{-2}$ when $0<x<r_{1}$.

Suppose we have constructed a product $B_{1} \cdots B_{k-1}$ whose behavior on $(0,1)$ reflects the behavior of the finite sequence $\left\{\gamma_{1}, \ldots, \gamma_{k-1}\right\}$.

If $\gamma_{k}<\gamma_{k-1}$, we construct the Blaschke product $B_{k}$ so that $B_{1} \cdots B_{k}$ has the radial limit $\gamma_{k}$ at 1 and so that $\left|B_{k}(x)-1\right|<2^{-k}$ on some interval $\left(0, r_{k-1}\right]$ on which the behavior of $B_{1} \cdots B_{k-1}$ also reflects the behavior of the finite sequence $\left\{\gamma_{1}, \ldots, \gamma_{k-1}\right\}$.

If $\gamma_{k}>\gamma_{k-1}$, we denote by $B_{k}$ the reciprocal of a Blaschke product whose zeros are zeros of $B_{1} \cdots B_{k-1}$ and whose radial limit at $z=1$ is $\gamma_{k-1} / \gamma_{k}$. To satisfy ourselves that the supply of zeros of $B_{1} \cdots B_{k-1}$ is sufficient for our purpose, we observe that $0<\gamma_{k}<1$ and that if we suppressed all except finitely many of the zeros of the function $B_{1} \cdots B_{k-1}$, the modulus of its radial limit at $z=1$ would rise to the value 1.

If for $k=1,2, \ldots$ the sequence of zeros (poles) of $B_{k}$ begins near enough to $C$, then the formal product $B=\Pi B_{k}$ is a Blaschke product and its radial cluster set at 1 is the segment $K$.

3. The unrestricted continuum. In this section, we suppose that $M=\{1\}$ and that $K_{1}=K$ is a nonempty continuum in $D \cup C$. Again we choose a sequence $\left\{\gamma_{n}\right\}$ whose set of limit points is the continuum $K$. In the statement of our technical conditions on the sequence $\left\{\gamma_{k}\right\}$, we replace $\gamma_{k}$ with $\left|\gamma_{k}\right|$ and impose the additional restriction that $\gamma_{k} / \gamma_{k+1} \rightarrow 1$ as $k \rightarrow \infty$. Repeating the construction described in $\S 2$, we obtain a Blaschke product $B$ whose behavior on the segment $[0,1)$ reflects with 
progressively higher accuracy the behavior of the sequence $\left\{\left|\gamma_{k}\right|\right\}$. We shall now show that we can also control the argument of $B$ on the segment $(0,1)$.

To determine the contribution to $\arg B(x)$ from a single Blaschke factor with its zero at $r e^{i \theta}(0<|\theta|<\pi)$, we write the factor in the form

$$
b(z)=e^{-i \theta}\left(r e^{i \theta}-z\right) /\left(1-r e^{-i \theta} z\right) .
$$

If the zero lies on the circle $\{|z-1 / 2|=1 / 2\}$, then $r=\cos \theta$, and therefore $b(0)=r=\cos \theta$ and

$$
b(1)=\frac{r-e^{-i \theta}}{1-r e^{-i \theta}}=\frac{i \sin \theta}{\sin ^{2} \theta+i \cos \theta \sin \theta}=e^{i \theta} .
$$

Because the function $b$ maps the segment $[0,1]$ on the real axis onto a circular arc orthogonal to $C$, and because this arc has the endpoints $\cos \theta$ and $e^{i \theta}$, the argument of $b(x)$ changes monotonically from 0 to $\theta$ as $x$ moves from 0 to 1 along the real axis.

Suppose we have constructed a finite product $B=B_{1} \cdots B_{n}$ (where each factor is either a subproduct of $B_{0}$ or the reciprocal of such a subproduct) with the following three properties. (i) The intersection of some disk $|z-1|<\delta_{n}$ with the set of zeros of $B$ is symmetric with respect to the real axis, in the strict sense that zeros at $z$ and at $\bar{z}$ have the same multiplicity. (ii) The zeros of $B_{0}$ in the upper half of the disk $|z-1|<\delta_{n}$ that occur in $B$ have indices forming a periodic sequence. (iii) The function $B$ maps the segment $[0,1)$ onto an arc that follows closely the polygonal arc with the vertices

$$
\gamma_{1}, \gamma_{2}, \ldots, \gamma_{n-1}, \gamma_{n-1}\left|\gamma_{n} / \gamma_{n-1}\right|
$$

From the discussion in the preceding paragraph it follows that the suppression of an additional zero $z_{k}$ changes the argument of the radial limit at 1 of $B$ by $-\arg z_{k}$. Because the arguments of the zeros (in the upper half-plane) of $B_{0}$ essentially form a harmonic sequence, we can choose a finite number of the surviving zeros of $B$ in the upper or lower half-plane so that after their suppression the function $B$ maps the segment $[1,0]$ onto an arc following closely the polygonal arc with the vertices $\gamma_{1}, \gamma_{2}, \ldots, \gamma_{n}$.

Next we adjoin to $B$ either a new Blaschke product $B_{n+1}$ (if $\left.\left|\gamma_{n+1}\right|<\left|\gamma_{n}\right|\right)$ or the reciprocal of a Blaschke product (if $\left.\left|\gamma_{n+1}\right|>\left|\gamma_{n}\right|\right)$. The continuation of the construction produces a Blaschke product whose radial cluster set at 1 is the continuum $K$.

4. The general case. If $M$ consists of a single point $\zeta$ on $C$ rather than the single point 1, we construct a Blaschke product $B$ having the radial cluster set $K$ at 1 and define a Blaschke product $P$ by the formula $P(z)=B(z / \zeta)$.

If $\left\{\zeta_{n}\right\}$ is a finite or countable set on $C$ and $\left\{K_{m}\right\}$ is a corresponding sequence of nonempty continua in $D \cup C$, we construct a sequence $\left\{P_{m}\right\}$ of Blaschke products such that for each index $m$ the function $P_{m}$ is holomorphic on $C$ (except at the point $\zeta_{m}$ ) and has at $\zeta_{m}$ the radial cluster set $K_{m}$.

If from each of the products $P_{m}$ we form a product $Q_{m}$ by suppressing a sufficiently large number of early zeros, the function $Q=\Pi Q_{m}$ is a Blaschke product with the property that for each index $m$ the subproduct $Q / Q_{m}$ has at $\zeta_{m}$ a 
radial limit of modulus 1 . The radial cluster set of $Q$ at $\zeta_{m}$ then has the form $e^{i \phi_{m}} K_{m}$, where $\phi_{m}$, the angle of rotation, depends not only on the radial limit at $\zeta$ of $Q / Q_{m}$, but also on the set of early zeros stripped from $P_{m}$ in the passage to $Q_{m}$. By Theorem 2 in [1], there exists a Blaschke product $Q_{0}$ whose radial limit at $\zeta_{m}$ is $e^{-i \phi_{m}}$ $(m=1,2, \ldots)$. Clearly, the Blaschke product $Q_{0} Q$ has the properties promised in our theorem.

\section{REFERENCES}

1. C. L. Belna, F. W. Carroll and G. Piranian, Strong Fatou-1-points of Blaschke products, Trans. Amer. Math. Soc. 280 (1983), 695-702.

2. G. T. Cargo, Blaschke products and singular functions with prescribed boundary values, J. Math. Anal. Appl. 71 (1979), 287-296.

Custom Courseware, P.O. BoX 434, Cicero, New York 13039

Department of Mathematics, Iowa State University, Ames, Iowa 50011

Department of Mathematics, University of Michigan, Ann ARBor, Michigan 48109 